\title{
Spin-polarized currents generated by magnetic Fe atomic chains
}

\author{
Zheng-Zhe Lin $^{1}$ and Xi Chen ${ }^{2}$ \\ ${ }^{1}$ School of Physics and Optoelectronic Engineering, Xidian University, Xi'an 710071, People's Republic \\ of China \\ ${ }^{2}$ Department of Applied Physics, School of Science, Xi'an Jiaotong University, Xi'an 710049, People's \\ Republic of China \\ E-mail: linzhengzhe@hotmail.com
}

Received 21 January 2014, revised 2 April 2014

Accepted for publication 16 April 2014

Published 21 May 2014

\begin{abstract}
Fe-based devices are widely used in spintronics because of high spin-polarization and magnetism. In this work, freestanding Fe atomic chains, the thinnest wires, were used to generate spin-polarized currents due to the spin-polarized energy bands. By $a b$ initio calculations, the zigzag structure was found to be more stable than the wide-angle zigzag structure and had a higher ratio of spin-up and spin-down currents. By our theoretical prediction, Fe atomic chains have a sufficiently long thermal lifetime only at $T \leqslant 150 \mathrm{~K}$, while $\mathrm{C}$ atomic chains are very stable even at $T=1000 \mathrm{~K}$. This means that the spintronic devices based on Fe chains could work only at low temperatures. A system constructed by a short Fe chain sandwiched between two graphene electrodes could be used as a spin-polarized current generator, while a $\mathrm{C}$ chain could not be used in this way. The present work may be instructive and meaningful to further practical applications based on recent technical developments on the preparation of metal atomic chains (Proc. Natl. Acad. Sci. USA 1079055 (2010)).
\end{abstract}

Keywords: spin-polarized current, atomic chain, quantum electron transport

(Some figures may appear in colour only in the online journal)

\section{Introduction}

Spintronics is a promising field for information processing, storage, and many other applications. In the past decade, much effort has been made in spintronics, and great developments have been seen [1-3]. In this area, generation and manipulation of spin-polarized current are two basic points, and minimization of spintronic devices is increasingly important. Since the discovery of graphene, more and more applications based on it have been proposed. Due to the halfmetallic nature for edge states in zigzag graphene nanoribbons $[4,5]$ - that is, metallic for electrons with one spin orientation and insulating for electrons with the other-graphene-based spin-filter devices were proposed. However, the edges of graphene suffer from spontaneous reconstruction $[6,7]$ or chemical passivation, which may change the halfmetallic electronic property. On the other hand, as the thinnest molecular wires, one-dimensional atomic chains have gained great attention for their possible spintronic applications. Over the past two decades, Au [8-11] and Pt [12, 13] atomic chains were prepared by pulling two contacted atom-sized metal junctions. In recent years, freestanding $\mathrm{C}$ chains were carved out from single-layer graphene with a high-energy electron beam [14], unraveled from sharp C specimens [15-17] or C nanotubes [18], and chemically synthesized [19-21]. Recently, much attention has been paid to metal atomic chains, and their stability has been studied [22, 23]. By elongating a $\mathrm{C}$ nanotube with an $\mathrm{Fe}$ nanorod inside, an $\mathrm{Fe}$ atomic chain clamped by a C nanotube was prepared [24], and the chemical reactivity and sensitivity of $\mathrm{Au}$ and $\mathrm{Ag}$ chains to small molecules were considered [25-27]. Among these atomic chains, magnetic chains may be the thinnest material used for generating and transporting spin-polarized currents because of high spin-polarization and magnetismthat is, Fe atomic chains [28]. In recent years, two-dimensional graphene was considered a good candidate for materials used in emerging electronics. In C-based circuits, onedimensional $\mathrm{C}$ chains are the thinnest natural wires, and an $\mathrm{Fe}$ 
chain embedded in a $\mathrm{C}$ chain may work with the circuits. $\mathrm{C}$ chains have been proven effective as spin-filters and spinvalves [29, 30], and a magnetic $\mathrm{Fe}$ chain embedded in a $\mathrm{C}$ chain should perform well on spin-polarized current generation. To design Fe-based low-dimensional spintronic devices, theoretical investigation on the thermal stability and electronic property of $\mathrm{Fe}$ chains should be beneficial to guild corresponding experiments.

In this work, the spintronic property of Fe atomic chains was studied. On this basis, a short Fe atomic chain clamped by graphene electrodes with $\mathrm{C}$ chains (figure 3(a)) was proposed as a spin-polarized current generator. To investigate the thermal stability of Fe atomic chains, a recently built statistical mechanical model [31-34] was employed. According to the result, the $\mathrm{C}-\mathrm{C}$ and $\mathrm{C}-\mathrm{Fe}$ bonds in atomic chains are very stable at room temperature, while Fe-Fe bonds could survive only below $150 \mathrm{~K}$ with a lifetime longer than $99 \mathrm{~h}$. A designed structure composed by graphene electrodes, C chains, and an $\mathrm{Fe}$ chain presented a feature of spin-polarized quantum electronic transport. The structure could be used as the smallest and thinnest spin-polarized current generator possible.

\section{Method}

To investigate the electronic properties of Fe atomic chains and corresponding structures, density functional theory calculations were performed using the SIESTA code [35]. The exchange-correlation functional was treated using a generalized gradient approximation according to Perdew-BurkeErnzerhof [36]. The norm-conserving Troullier-Martins pseudopotentials [37] were used to describe the core electrons. For structure optimization, the double- $\zeta$ plus polarization basis sets were used, and the grid mesh cutoff was set at $150 \mathrm{Ry}$. The structures were relaxed until the atomic forces became less than $0.01 \mathrm{eV} \AA^{-1}$. For molecular dynamics (MD) simulations, a non-self-consistent Harris functional was used to save computation time.

For quantum transport, calculations were performed using the TRANSIESTA module [38]. For a bias voltage $V_{b}$, the spin-up part $I_{+}$and spin-down part $I_{-}$of the current is given by the Landauer-Buttiker formula [39]:

$$
\begin{aligned}
I_{ \pm}= & \frac{2 e}{h} \int T_{ \pm}\left(E, V_{b}\right)\left[f_{L}\left(E-E_{F}-\frac{e V_{b}}{2}\right)\right. \\
& \left.-f_{R}\left(E-E_{F}+\frac{e V_{b}}{2}\right)\right] d E .
\end{aligned}
$$

Here, $T_{+}\left(E, V_{\mathrm{b}}\right)$ and $T_{-}\left(E, V_{\mathrm{b}}\right)$ are the transmission rates of spin-up and spin-down electrons at energy $E$, respectively. $E_{F}$ is the Fermi energy of the electrodes. $f_{L}$ and $f_{R}$ are the Fermi-Dirac distribution functions for both electrodes, respectively. To save computation time, the single- $\zeta$ plus polarization basis sets were used.

A simple statistical mechanical model [31-34] built previously was applied to predict the lifetime of chemical bonds in atomic chains at different temperatures. In atomic chains, an element process may involve a transfer of a 'key atom' in a potential valley crossing over $E_{0}$. In most cases, the atomic kinetic energy $\varepsilon$ at the valley bottom is significantly smaller than $E_{0}$, and the atom vibrates many times in the valley before crossing over the barrier. Based on the Boltzmann distribution, the probability $P$ of the atomic kinetic energy $\varepsilon$ larger than $E_{0}$ reads:

$$
P=\frac{\int_{E_{0}}^{+\infty} \varepsilon^{1 / 2} e^{-\varepsilon / k_{B} T} d \varepsilon}{\int_{0}^{+\infty} \varepsilon^{1 / 2} e^{-\varepsilon / k_{B} T} d \varepsilon}=\frac{\int_{E_{0}}^{+\infty} \varepsilon^{1 / 2} e^{-\varepsilon / k_{B} T} d \varepsilon}{\sqrt{\pi}\left(k_{B} T\right)^{3 / 2} / 2},
$$

where $\varepsilon^{1 / 2} e^{-\varepsilon / k_{B} T}$ is the Boltzmann distribution and $\int_{0}^{+\infty} \varepsilon^{1 / 2} e^{-\varepsilon / k_{B} T} d \varepsilon$ is the partition function. Then, the atomic transfer rate $k_{1 s t}$ over the barrier reads:

$$
k_{1 s t}=k_{0} \frac{\int_{E_{0}}^{+\infty} \varepsilon^{1 / 2} e^{-\varepsilon / k_{B} T} d \varepsilon}{\sqrt{\pi}\left(k_{B} T\right)^{3 / 2} / 2},
$$

and the thermal lifetime of the chemical bond is $\tau=1 / k_{1 s t}$. Here, $k_{0}$ is the attempt frequency of the key atom vibrating in the potential valley, which can be evaluated by the potential energy $U=U(s)$ along the reaction path with $d s=\left(\sum_{i=1}^{n} m_{i} d \vec{r}_{i}^{2}\right)^{1 / 2}$ as the reaction coordinate. The Lagrangian along the reaction path is:

$$
L=\frac{1}{2}\left(\frac{d s}{d t}\right)^{2}-U
$$

and the corresponding Lagrange's equation approximately reads:

$$
\frac{d^{2} s}{d t^{2}}+k_{0}^{2} s=0
$$

where $k_{0}=\left.\frac{d^{2} U}{d s^{2}}\right|_{s=0}$ is just the attempt frequency. In our previous work, this model was widely verified by reaction rate data from MD simulations [31-34].

\section{Results and discussion}

\subsection{General information of infinite Fe atomic chains}

To get basic information, geometry optimizations and energy band calculations were performed for infinite $\mathrm{Fe}$ atomic chains. Linear and zigzag Fe chains were taken into account as possible structures, and considering the Peierls distortion, a unit cell with two atoms was used. According to the result, the zigzag structure (upper figure 1(a)) has the lowest potential energy, and with the Peierls distortion, the corresponding bond lengths are $c_{1}=2.37 \AA, c_{2}=2.55 \AA$, and $c=2.62 \AA$. The cohesive energy and the magnetic moment per unit cell are $E_{\mathrm{c}}=2.64 \mathrm{eV} /$ atom and $\mu=3.80 \mu_{\mathrm{B}}$, respectively. The above result is close to reference [40] $\left(c_{1}=2.24 \AA, c_{2}=2.42 \AA\right.$, $c=2.40 \AA, E_{\mathrm{c}}=2.69 \mathrm{eV} /$ atom, and $\mu=3.19 \mu_{\mathrm{B}}$, by ultrasoft pseudopotentials and the plane-wave basic set). It is worth 
(a)

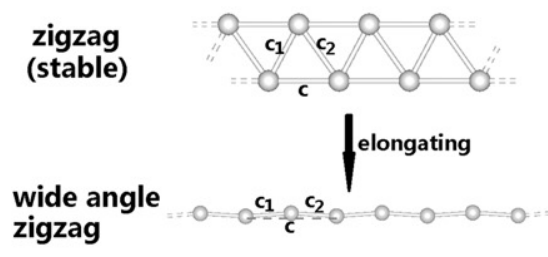

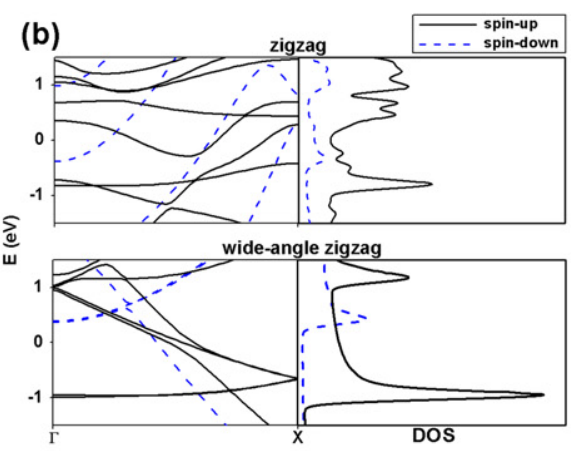

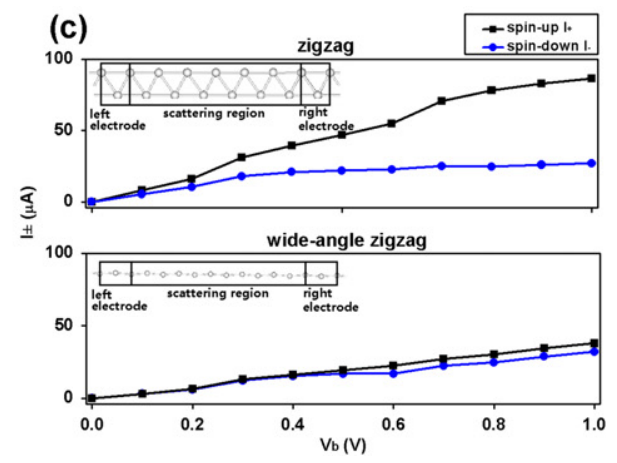

Figure 1. (a) The zigzag structure of an infinite Fe atomic chain (upper) and the wide-angle zigzag structure (lower) that is elongated from the zigzag one. (b) The energy bands and DOS of zigzag (upper) and wide-angle zigzag (lower) Fe atomic chains, where $\Gamma$ is the center and $\mathrm{X}$ is the boundary of the Brillouin zone. (c) The spin-up and spin-down current $I_{+}$and $I_{-}$at different bias $V_{\mathrm{b}}$ for zigzag (upper) and wide-angle zigzag (lower) Fe atomic chains.

noting that in some papers the Fe chain was simply considered as a linear atomic chain $[28,41,42]$, which may be not the most stable structure. Figure 1(b) (upper) shows the energy band profile and the density of state (DOS) for the zigzag $\mathrm{Fe}$ atomic chain, presenting a spin-polarized feature. To investigate the quantum transport property, a model was built by one and six unit cells of a zigzag Fe chain selected as the electrode and the scattering region, respectively (figure $1(\mathrm{c})$ ). A $1 \times 1 \times 100 k$-point sampling was used for the electrodes, and a vacuum layer of $20 \AA$ was used along $x$ and $y$ directions to minimize the interaction between neighboring unit cells. The result (upper figure 1(c)) indicates that the current is spin-polarized and $I_{+}: I_{-}=3.2$ at $V_{\mathrm{b}}=1.0 \mathrm{~V}$. In the range of $V_{\mathrm{b}}=0.0 \sim 1.0 \mathrm{~V}$, the average conductances for spinup and spin-down electrons are about 1.11 and $0.35 \mathrm{G}_{0}$ $\left(\mathrm{G}_{0}=2 e^{2} / h\right.$ is the quantum conductance), respectively, which is close to the values in reference [43].

In an experimental manipulation of nanostructures such as mechanically controllable break junctions [8-13], an atomic chain can be elongated. To investigate the effect of elongation on an Fe chain, we enlarged the lattice constant $c$ of a zigzag Fe chain step by step and performed geometry optimizations. According to the result, another local minima of potential energy was found at $c=4.68 \AA$, which is elongated from a zigzag $\mathrm{Fe}$ chain and can be called a wide-angle zigzag Fe chain (lower figure 1(a)). This stable structure has a smaller cohesive energy $E_{\mathrm{c}}=1.77 \mathrm{eV} /$ atom than the zigzag structure and a magnetic moment $\mu=3.57 \mu_{\mathrm{B}}$ per unit cell. Figure 1(b) (lower) shows the energy band profile and DOS of the wide-angle zigzag Fe atomic chain, presenting a spin-polarized feature. For quantum transport, the current is less spin-polarized than that of the zigzag chain (lower figure 1(c)), with a ratio of $I_{+}: I_{-}=1.2$ at $V_{\mathrm{b}}=1.0 \mathrm{~V}$. In the range of $V_{\mathrm{b}}=0.0 \sim 1.0 \mathrm{~V}$, the average conductances for spin-up and spin-down electrons are about 0.49 and $0.42 \mathrm{G}_{0}$, respectively.

\subsection{Thermal lifetime of $C_{8}, C_{4} F e C_{4}$, and $C_{4} F_{4} C_{4}$ chains}

The stability of $\mathrm{Fe}$ atomic chains is vitally important in practical applications and must be investigated. Recently, a theoretical model was proposed to formulate criteria for the producibility of freestanding metal atomic chains in mechanically controllable break junctions [22], and the producibility of C atomic chains was also studied [44, 45]. Here, we consider the thermal lifetime of $\mathrm{Fe}$ chains connected with $\mathrm{C}$ chainsthat is, $\mathrm{C}_{8}, \mathrm{C}_{4} \mathrm{FeC}_{4}$, and $\mathrm{C}_{4} \mathrm{Fe}_{4} \mathrm{C}_{4}$ chains (figure 2(a)). In thermal motion, a chemical bond in the chain may break when two neighboring atoms move away from each other (e.g., the progress shown in figure 2(a)), and the average lifetime of the bond is $\tau=1 / k_{1 \mathrm{st}}$, where $k_{1 \mathrm{st}}$ can be evaluated by equations (3) and (5). To apply equations (3) and (5), the potential profiles along the minimum energy paths (MEPs) for bond breaking of the $\mathrm{C}-\mathrm{C}$ in the $\mathrm{C}_{8}$ chain, the $\mathrm{C}-\mathrm{Fe}$ in the $\mathrm{C}_{4} \mathrm{FeC}_{4}$ chain, and the middle $\mathrm{Fe}-\mathrm{Fe}$ in the $\mathrm{C}_{4} \mathrm{Fe}_{4} \mathrm{C}_{4}$ chain (figure 2(b)) were calculated with two terminal chain atoms fixed. As an example, the MEP for bond breaking of the middle Fe-Fe bond in the $\mathrm{C}_{4} \mathrm{Fe}_{4} \mathrm{C}_{4}$ chain is shown in figure 1(a). For the above bondbreaking processes, we got $E_{0}=5.44,5.17$, and $0.51 \mathrm{eV}$ and $k_{0}=8.05 \times 10^{11}, 1.93 \times 10^{11}$, and $5.75 \times 10^{10} \mathrm{~s}^{-1}$ (by equation (5)), respectively. Then, by equation (3), the average lifetime $\tau$ of the bonds at different temperatures was derived (figure 2(c)). According to equations (3) and (5), the middle $\mathrm{C}-\mathrm{C}$ bond in the $\mathrm{C}_{8}$ chain has a lifetime of about $6 \times 10^{70}$ years at $T=300 \mathrm{~K}$. Even at $T=1000 \mathrm{~K}$, this bond still has a lifetime of about $1 \times 10^{7}$ years. For the $\mathrm{C}$-Fe bond in the $\mathrm{C}_{4} \mathrm{FeC}_{4}$ chain, the lifetime is about $6 \times 10^{66}$ and $2 \times 10^{6}$ years at $T=300$ and $1000 \mathrm{~K}$, respectively. The result indicates that the pure and Fedoped $\mathrm{C}$ chains are very stable. However, at $T=300 \mathrm{~K}$, the middle $\mathrm{Fe}$-Fe bond in the $\mathrm{C}_{4} \mathrm{Fe}_{4} \mathrm{C}_{4}$ chain has a lifetime of only $1.30 \mathrm{~ms}$. Fortunately, at $T \leqslant 150 \mathrm{~K}$, the lifetime is predicted to be longer than $99 \mathrm{~h}$. By ab initio MD simulation, we found that at $T=300 \mathrm{~K}$, the middle $\mathrm{Fe}$-Fe bond of the $\mathrm{C}_{4} \mathrm{Fe}_{4} \mathrm{C}_{4}$ chain kept stable even in a time of $50 \mathrm{ps}$, while it survived for only $0.2 \mathrm{ps}$ at $T=1000 \mathrm{~K}$. This result is generally in accordance with the above theoretical prediction. For this reason, $\mathrm{Fe}$ atomic chains may serve as spintronic devices at low temperatures.

\subsection{Spin-polarized electronic transport}

Based on optimized structures, we investigated the spintronic properties of an $\mathrm{Fe}$ atomic chain coupled with a $\mathrm{C}$ chain, which is the thinnest magnetic wire in a graphene-based 

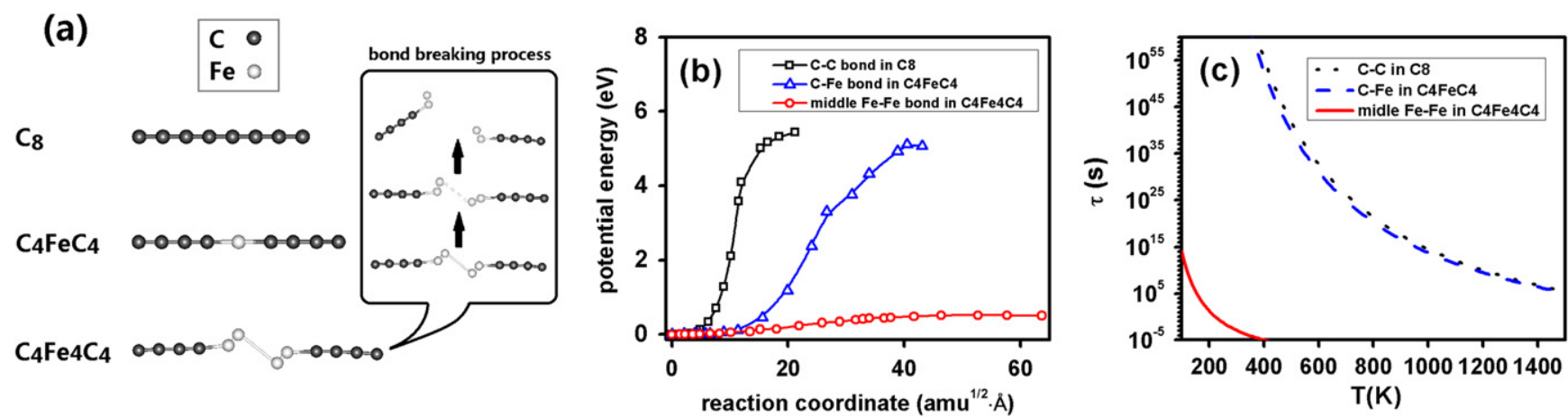

Figure 2. (a) The structures of $\mathrm{C}_{8}, \mathrm{C}_{4} \mathrm{FeC}_{4}$, and $\mathrm{C}_{4} \mathrm{Fe}_{4} \mathrm{C}_{4}$ atomic chains. The right panel shows some snapshots along the MEP for bond breaking of the middle $\mathrm{Fe}-\mathrm{Fe}$ bond. (b) Potential energy profile along the MEP for bond breaking of $\mathrm{C}-\mathrm{C}$ in the $\mathrm{C}_{8}$ chain, $\mathrm{C}-\mathrm{Fe}$ in the $\mathrm{C}_{4} \mathrm{FeC} \mathrm{C}_{4}$ chain, and middle $\mathrm{Fe}-\mathrm{Fe}$ in the $\mathrm{C}_{4} \mathrm{Fe}_{4} \mathrm{C}_{4}$ chain. (c) The average lifetime $\tau=1 / k_{1 \mathrm{st}}$ of these three bonds changing with temperature $T$.
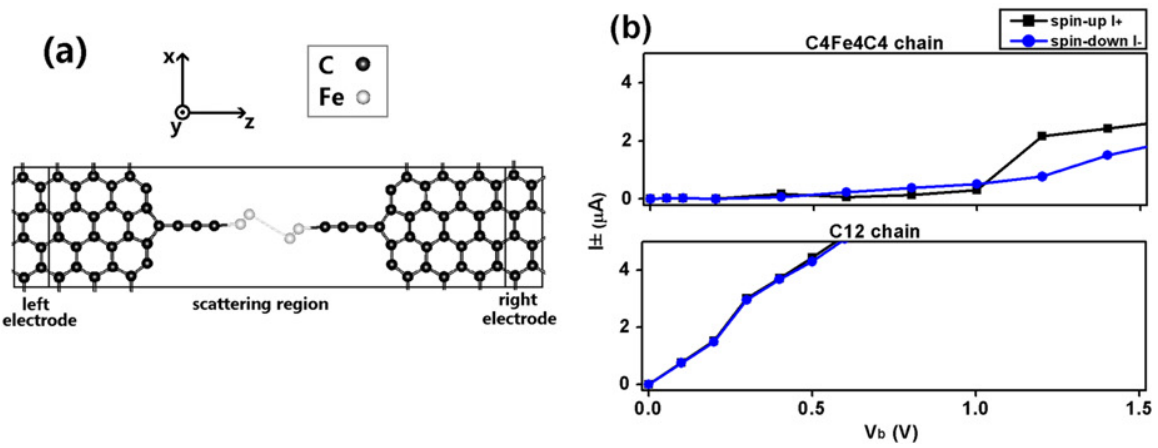

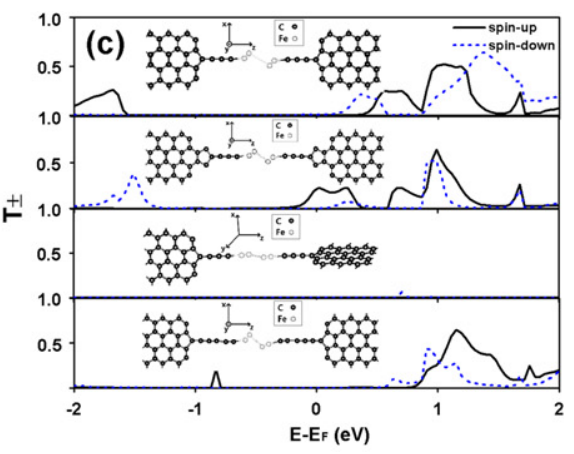

Figure 3. (a) A two-probe system constructed by a $\mathrm{C}_{4} \mathrm{Fe}_{4} \mathrm{C}_{4}$ atomic chain as the scattering region sandwiched between two semi-infinite graphene electrodes. Periodic boundary conditions are shown by the solid frame. (b) The spin-up and spin-down current $I_{+}$and $I_{-}$at a different bias $V_{\mathrm{b}}$ for $\mathrm{a}_{4} \mathrm{Fe}_{4} \mathrm{C}_{4}$ (upper) chain and a $\mathrm{C}_{12}$ (lower) chain sandwiched between graphene electrodes. (c) Transmission spectra at $V_{\mathrm{b}}=0 \mathrm{~V}$ for four two-probe systems including a C-Fe-C atomic chain. Corresponding scattering regions are shown in each subfigure. The solid and dashed lines indicate $T_{+}$and $T_{-}$for spin-up and spin-down electrons, respectively.

circuit. A two-probe system, with a $\mathrm{C}_{4} \mathrm{Fe}_{4} \mathrm{C}_{4}$ atomic chain as the scattering region sandwiched between two semi-infinite graphene electrodes, was constructed along the $z$ direction (figure 3(a)). Periodic boundary conditions are imposed on the electrodes (on the $x$ and $z$ directions) and the scattering region (on the $x$ direction), as shown by the solid frame. Different lengths of buffer layers in the scattering region were tested to investigate the influence between the two electrodes, and three unit cells of the electrode were found to be enough. The zigzag direction of graphene electrodes was chosen as the $z$ direction, because the $k$ vectors of the electronic states near the Fermi energy - that is, on the Dirac point — are along the $z$ direction, and thus the conductivity of the zigzag direction is better than the armchair direction [46]. A $4 \times 1 \times 100 k$-point sampling was used for the electrodes. The vacuum layer along the $y$ direction is $20 \AA$ to minimize the interaction between neighboring sheets.

According to the result (upper figure 3(b)), for $V_{\mathrm{b}}=0.6 \sim 1.0 \mathrm{~V}$, the spin-down current $I_{-}$is larger than the spin-up current $I$, presenting a spin-polarized feature. The ratio is $I_{-}: I_{+}=3.8$ at $V_{\mathrm{b}}=0.6 \mathrm{~V}$, and it decreases to $I_{-}: I_{+}=1.7$ at $V_{\mathrm{b}}=1.0 \mathrm{~V}$. However, in this voltage range the current is quite small, and the average conductances for spin-up and spin-down electrons are only 0.008 and $0.009 \mathrm{G}_{0}$, respectively. For $V_{\mathrm{b}}>1.0 \mathrm{~V}$, the current is larger than that for
$V_{\mathrm{b}}=0.6 \sim 1.0 \mathrm{~V}$, and the spin-up current $I_{+}$is obviously larger than the spin-down current $I_{-}$. At $V_{\mathrm{b}}=1.4 \mathrm{~V}$, the ratio $I_{+}$: $I_{-}=1.6$. By comparison, at the same $V_{\mathrm{b}}, I_{+}$and $I_{-}$of this system are in an order of magnitude smaller than those of an infinite $\mathrm{Fe}$ atomic chain. In the range of $V_{\mathrm{b}}=1.0 \sim 1.4 \mathrm{~V}$, the average conductances for spin-up and spin-down electrons are about 0.05 and $0.03 \mathrm{G}_{0}$, respectively. To investigate the mechanism of spin-polarized currents, the transmission spectrum at $V_{b}=0 \mathrm{~V}$ for the two-probe system in figure 3(a) is plotted in the first subfigure of figure 3(c). Transmission peaks for spin-up and spin-down electrons can be found at different energy $E$. These peaks should correspond to molecular electronic states of the scattering region. In the range of $E-E_{F}=0 \sim 1 \mathrm{eV}$, a spin-down transmission peak locates at an energy range lower than a spin-up peak. Therefore, with increasing $V_{b}$, the spin-down current $I_{-}$is first larger and then smaller than the spin-up current $I_{+}$. In the range of $E-E_{F}>1 \mathrm{eV}$, another spin-up transmission peak appears with higher transmission than the spin-down peak in this range, and correspondingly for $V_{\mathrm{b}}>1.0 \mathrm{~V}, I_{+}$is larger than $I_{-}$.

To investigate the influence of different shapes of electrode ends or lengths of a $\mathrm{C}$ chain on the spin-polarized currents, three other structures (shown in the second to fourth subfigures of figure 3(c)) were considered, which consist of 
the one with tapered electrode ends, the one whose electrode planes are perpendicular to each other, and the one with longer $\mathrm{C}$ chains, respectively. For the structure with tapered electrode ends, the transmission of spin-up electrons is always larger than spin-down electrons in the range of $E-E_{F}=0 \sim 2$ $\mathrm{eV}$, indicating that the currents are spin-polarized. For the structure whose electrode planes are perpendicular to each other, the transmission of spin-up and spin-down electrons is nearly zero in the whole energy range-that is, the conductance is very low at any $V_{b}$. This is because in this case the $p-\pi$ conjugated orbitals in graphene electrodes are orthogonal to each side and then the transmission is forbidden. It can be inferred that when the two electrodes have an angle $\theta$, the transmission $T_{ \pm}$is proportional to $\cos ^{2} \theta$. For the structure with longer $\mathrm{C}$ chains, the transmission of spin-up electrons is also larger than spin-down electrons in the range of $E$ $E_{F}>1 \mathrm{eV}$. In summary, for $V_{b}>1 \mathrm{eV}$, the spin-polarized currents by the $\mathrm{Fe}$ atomic chain are independent of the geometry of electrodes or $\mathrm{C}$ chains but are weakened by the increase in angle between the two electrodes.

Finally, to make a comparison with a pure $\mathrm{C}$ atomic chain, we replaced four $\mathrm{Fe}$ atoms with four $\mathrm{C}$ atoms, and $I_{+}$ and $I_{-}$of the optimized structure are shown in lower figure 3(b). It can be seen that the pure $\mathrm{C}$ chain exhibits the typical characteristics of spin-non-polarized conductors, with a conductance of about $0.11 \mathrm{G}_{0}$ for spin-up and spin-down electrons. According to the above results, $\mathrm{Fe}$ atomic wire could act as a spin-polarized current generator in C-based circuits.

\section{Conclusions}

Through the above theoretical investigation, freestanding $\mathrm{Fe}$ atomic chains were proposed to be used as the thinnest wire to generate spin-polarized currents. A long Fe chain has a stable zigzag structure and a spin-polarized energy band. At a bias of $1.0 \mathrm{~V}$, the ratio of spin-up and spin-down current in a zigzag $\mathrm{Fe}$ chain is about $3.2: 1$. When a zigzag Fe chain is elongated, it transforms into a wide-angle zigzag structure, whose ratio of spin-up and spin-down current is lower than that of a zigzag structure $(1.2: 1$ at $1.0 \mathrm{~V})$. By our theoretical prediction, $\mathrm{Fe}$ atomic chains have sufficiently long thermal lifetimes only at $T \leqslant 150 \mathrm{~K}$, while $\mathrm{C}$ atomic chains are very stable even at $T=1000 \mathrm{~K}$. This result means that the spintronic devices based on Fe chains could work only at low temperatures. A short $\mathrm{Fe}$ chain sandwiched between two graphene electrodes was taken as an example for spintronic devices, and the quantum electronic transport was investigated. At a certain bias, the currents through this system are spin-polarized, while a similar property was not found for pure $\mathrm{C}$ chains. By recently developed techniques for preparing $\mathrm{Fe}$ chains clamped by $\mathrm{C}$ nanotubes [24], the spintronic property of $\mathrm{Fe}$ chains may be put into future practical applications.

\section{Acknowledgments}

This work was supported by the National Natural Science Foundation of China under Grant No. 11304239, and the Fundamental Research Funds for the Central Universities.

\section{References}

[1] Wolf S, Awschalom D, Buhrman R, Daughton J, Von Molnar S, Roukes M, Chtchelkanova A and Treger D 2001 Science 2941488

[2] Zutic I, Fabian J and Das Sarma S 2004 Rev. Mod. Phys. 76323

[3] Fert A 2008 Rev. Mod. Phys. 801517

[4] Son Y-W, Cohen M L and Louie S G 2006 Nature 444347

[5] Saffarzadeh A and Farghadan R 2011 Appl. Phys. Lett. 98023106

[6] Koskinen P, Malola S and Häkkinen H 2009 Phys. Rev. B 80 073401

[7] Koskinen P, Malola S and Häkkinen H 2008 Phys. Rev. Lett. 101115502

[8] Ohnishi H, Kondo Y and Takayanagi K 1998 Nature 395780

[9] Yanson A I, Bollinger G R, van den Brom H E, Agraït N and van Ruitenbeek J M 1998 Nature 395783

[10] Kizuka T 1998 Phys. Rev. Lett. 814448

[11] Rodrigues V, Fuhrer T and Ugarte D 2000 Phys. Rev. Lett. 854124

[12] Krans J M, Muller C J, Yanson I K, Govaert T C M, Hesper R and van Ruitenbeek J M 1993 Phys. Rev. B 48 14721

[13] Kizuka T and Monna K 2009 Phys. Rev. B 80205406

[14] Jin C, Lan H, Peng L, Suenaga K and Iijima S 2009 Phys. Rev. Lett. 102205501

[15] Mikhailovskij I M, Wanderka N, Ksenofontov V A, Mazilova T I, Sadanov E V and Velicodnaja O A 2007 Nanotech. 18475705

[16] Mazilova T I, Mikhailovskij I M, Ksenofontov V A and Sadanov E V 2009 Nano Lett. 9774

[17] Mikhailovskij I M, Sadanov E V, Mazilova T I and Velicodnaja O A 2009 Phys. Rev. B 80165404

[18] Börrnert F et al 2010 Phys. Rev. B 81085439

[19] Eisler S, Slepkov A D, Elliott E, Luu T, McDonald R, Hegmann F A and Tykwinski R R 2005 J. Am. Chem. Soc. 1272666

[20] Chalifoux W A, McDonald R, Ferguson M J and Tykwinski R R 2009 Angew. Chem. Int. Edit. 487915

[21] Chalifoux W A and Tykwinski R R 2010 Nat. Chem. 2967

[22] Thiess A, Mokrousov Y, Blügel S and Heinze S 2008 Nano Lett. 82144

[23] Zaki N, Marianetti C A, Acharya D P, Zahl P, Sutter P, Okamoto J, Johnson P D, Millis A J and Osgood R M 2013 Phys. Rev. B 87 161406(R)

[24] Tang D-M, Yin L-C, Li F, Liu C, Yu W-J, Hou P-X, Wu B, Lee Y-H, Ma X-L and Cheng H-M 2010 Proc. Natl. Acad. Sci. USA 1079055

[25] Ishida H 2007 Phys. Rev. B 75205419

[26] Sclauzero G, Dal Corso A and Smogunov A 2012 Phys. Rev. B 85165411

[27] Sclauzero G and Corso A D 2013 Phys. Rev. B 87085108

[28] Dalgleish H and Kirczenow G 2005 Phys. Rev. B 72155429

[29] Zeng M G, Shen L, Cai Y Q, Sha Z D and Feng Y P 2010 Appl. Phys. Lett. 96042104

[30] Xu Y, Wang B-J, Ke S-H, Yang W and Alzahrani A Z 2012 J. Chem. Phys. 137104107

[31] Lin Z-Z and Ning X J 2011 EPL 9547012

[32] Lin Z-Z and Chen X 2013 EPL 10148002

[33] Yu W-F, Lin Z-Z and Ning X-J 2013 Phys. Rev. E 87062311

[34] Yu W-F, Lin Z-Z and Ning X-J 2013 Chin. Phys. B 22116802 
[35] Soler J M, Artacho E, Gale J D, García A, Junquera J, Ordejón P and Sánchez-Portal D 2002 J. Phys.: Condens. Matter 142745

[36] Perdew J P, Burke K and Ernzerhof M 1996 Phys. Rev. Lett. 773865

[37] Troullier N and Martins J L 1991 Phys. Rev. B 431993

[38] Brandbyge M, Mozos J L, Ordejón P, Taylor J and Stokbro K 2002 Phys. Rev. B 65165401

[39] Büttiker M, Imry Y, Landauer R and Pinhas S 1985 Phys. Rev. B 316207

[40] Ataca C, Cahangirov S, Durgun E, Jang Y-R and Ciraci S 2008 Phys. Rev. B 77214413
[41] Smogunov A, Dal Corso A and Tosatti E 2004 Phys. Rev. B 70 045417

[42] Autès G, Barreteau C, Spanjaard D and Desjonquères M-C 2008 Phys. Rev. B 77155437

[43] Velev J and Butler W H 2004 Phys. Rev. B 69094425

[44] Wang Y, Ning X-J, Lin Z-Z, Li P and Zhuang J 2007 Phys. Rev. B 76165423

[45] Wang Y, Lin Z-Z, Zhang W, Zhuang J and Ning X-J 2009 Phys. Rev. B 80233403

[46] Shen L, Zeng M G, Yang S-W, Zhang C, Wang X and Feng Y P 2010 J. Am. Chem. Soc. 13211481 